АССОЦИАЦИИ ПОЛИМОРФИЗМОВ ГЕНОВ ЦИТОКИНОВ И МАТРИКСНЫХ

МЕТАЛЛОПРОТЕИНАЗ С МИНЕРАЛЬНОЙ ПЛОТНОСТЬЮ КОСТНОЙ ТКАНИ

У ЖЕНЩИН В ПОСТМЕНОПАУЗЕ С САХАРНЫМ ДИАБЕТОМ 2 ТИПА

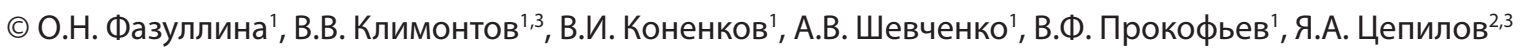

'Научно-исследовательский институт клинической и экспериментальной лимфологии» - филиал ФГБНУ «Федеральный исследовательский центр Институт цитологии и генетики Сибирского отделения Российской академии наук», Новосибирск

ФГБНУ «Федеральный исследовательский центр Институт цитологии и генетики Сибирского отделения Российской академии наук», Новосибирск

ЗФГАОУ ВО Новосибирский национальный исследовательский государственный университет, Новосибирск

ОБОСНОВАНИЕ. Остеопороз и сахарный диабет 2 типа (СД2) - частые коморбидные состояния у женщин в постменопаузе. Важную роль в ремоделировании костей в период постменопаузы могут играть цитокины и матриксные металлопротеиназы (MMPs). Показано, что аллельные варианты в полиморфных позициях генов цитокинов и ММРs влияют на экспрессию продуктов данных генов в нормальных и патологических условиях.

ЦЕЛЬ. Определить ассоциации вариантов генотипов в промоторах генов цитокинов (TNFA, IL1B, IL4, IL6, IL10, VEGFA) и MMPs (MMP2, MMP3, MMP9) с минеральной плотностью костной ткани (МПК) у женщин с СД2, находящихся в постменопаузе.

МАТЕРИАЛЫ И МЕТОДЫ. Обследовано 197 женщин с СД2, европеоидного происхождения, от 50 до 70 лет. Исследование МПК в позвоночнике, проксимальных отделах бедра и предплечье осуществляли с помощью двухэнергетической рентгеновской абсорбциометрии. Исследовано 13 точек полиморфизма, локализованных в промоторных регионах генов TNFA: -238 A/G (rs361525), -308 A/G (rs1800629) и -863 C/A (rs1800630), IL1B:-31 C/T (rs1143627), IL4:-590 C/T (rs2243250), IL6:-174 C/G (rs1800795), IL10:-592 C/A (rs1800872) и -1082 A/G (rs1800896), VEGFA: -2578 C/A (rs699947) и +936 C/T (rs3025039), MMP2: -1306 C/T (rs243865), MMP3:-1171 5A/6A (rs3025058), MMP9: -1562 C/T (rs3918242).

РЕЗУЛЬТАТЫ. Семьдесят три женщины имели нормальную МПК, 90 - остеопению, 34 - остеопороз. Возраст, ИМТ и курение были наиболее значимыми предикторами МПК в многофакторном регрессионном анализе ( $<<0,0001, \mathrm{p}=0,003$ и $\mathrm{p}=0,01$ соответственно). В аддитивной модели аллель С и генотип СС в позиции -1562 ММР9 были ассоциированы с низкой МПК (ОШ 2,16, 95\% ДИ 1,29-3,6, p=0,0007 и ОШ 2,02, 95\% ДИ 1,08-3,79, p=0,0008 соответственно). Ассоциация полиморфизма в гене MMP9 с МПК оставалась значимой после учета клинических факторов риска (р<0,001). При биоинформационном анализе выявлено 12 комбинаций генотипов, положительно ассоциированных с низкой МПК (все р <0,005). В составе этих комбинаций наиболее часто встречался гомозиготный генотип СС в позиции -1562 MMP9, генотип CС в позиции -863 TNFA, генотип GG в позиции -308 TNFA и генотип AA в позиции -1082 IL 10.

ЗАКЛЮЧЕНИЕ. Вариабельность в промоторах генов цитокинов и MMPs может вносить вклад в предрасположенность к остеопорозу у женщин с СД2, находящихся в постменопаузе.

КЛЮЧЕВЫЕ СЛОВА: сахарный диабет; остеопороз; постменопауза; плотность костной ткани; генетический полиморфизм

\title{
ASSOCIATIONS OF POLYMORPHISMS IN THE GENE PROMOTERS OF CYTOKINES AND MATRIX METALLOPROTEINASES WITH BONE MINERAL DENSITY IN POSTMENOPAUSAL TYPE 2 DIABETIC WOMEN
}

(c) Olga N. Fazullina' ${ }^{1}$, Vadim V. Klimontov,3, Vladimir I. Konenkov' ${ }^{1}$, Alla V. Shevchenko ${ }^{1}$, Viktor F. Prokofiev ${ }^{1}$, Yakov A. Tsepilov 2,3

${ }^{1}$ Research Institute of Clinical and Experimental Lymphology - Branch of the Institute of Cytology and Genetics, Siberian Branch of Russian Academy of Sciences, Novosibirsk, Russia

${ }^{2}$ Institute of Cytology and Genetics, Siberian Branch of Russian Academy of Sciences, Novosibirsk, Russia

${ }^{3}$ Novosibirsk State University, Novosibirsk, Russia

BACKGROUND. Osteoporosis and type 2 diabetes are common comorbidities in postmenopausal women. An important role in the bone remodeling over the menopausal transition can be played by cytokines and matrix metalloproteinases (MMPs). It was shown that allelic variants in polymorphic positions of the genes of cytokines and MMPs affect the expression of these molecules under normal and pathological conditions. 
AIMS. To examine associations between polymorphisms in the gene promoters of cytokines (TNFA, IL1B, IL4, IL6, IL 10, VEGFA) and MMPs (MMP2, MMP3, MMP9) with bone mineral density (BMD) in postmenopausal women with type 2 diabetes.

MATERIALS AND METHODS. We studied 197 Caucasian diabetic women, from 50 to70 years of age. An examination of BMD in the spine, proximal femur and forearm was performed by DEXA. Thirteen polymorphisms in the promoters of TNFA: -238 $A / G$ (rs361525), -308 A/G (rs1800629) and -863 C/A (rs1800630), IL1B: -31 C/T (rs1143627), IL4: -590 C/T (rs2243250), IL6: -174 C/G (rs1800795), IL10:-592 C/A (rs1800872) and -1082 A/G (rs1800896), VEGFA:-2578 C/A (rs699947) and +936 C/T (rs3025039), MMP2:-1306 C/T (rs243865), MMP3: - 1171 5A/6A (rs3025058) and MMP9:-1562 C/T (rs3918242), were investigated.

RESULTS. Seventy-three women had normal BMD, in 90 ones we revealed osteopenia, and 34 women had osteoporosis. Age, $B M I$ and smoking were strongest predictors of $B M D$ in multivariate regression analysis $(p<0.0001, p=0.003$ and $p=0.01$, respectively). In the additive model, $C$ allele and CC genotype in MMP9 -1562 position were associated with low BMD (OR 2.16, $p=0.0007$ and OR 2.02, $p=0.0008$, respectively). Association of the polymorphism with BMD remained significant after adjustment for clinical risk factors $(\mathrm{p}<0.001)$. Twelve combinations of genotypes, associated positively with low BMD, were revealed by bioinformatic analysis (all $\mathrm{p}<0.005$ ). The CC genotype in position -1562 of MMP9, CC genotype in position -863 of TNFA, GG genotype in position -308 of TNFA, and AA genotype in position -1082 of IL 10 were the most prevalent variants in these combinations.

CONCLUSIONS. Variability in the gene promoters of cytokines and MMPs may confer individual susceptibility to osteoporosis in postmenopausal type 2 diabetic women.

KEYWORDS: diabetes mellitus; osteoporosis; postmenopause; bone density; genetic polymorphism

Значительная часть заболеваний, встречающихся в практике эндокринолога, может осложняться развитием остеопороза [1]. Все более частыми коморбидными состояниями становятся сахарный диабет 2 типа (СД2) и остеопороз [2]. Установлено, что пациенты с СД2 имеют повышенный риск переломов бедра, позвоночника, плеча и предплечья в сравнении с лицами без СД [3, 4], даже при равных показателях минеральной плотности костной ткани (МПК) и индекса Fracture Risk Assessment Tool (FRAX) [5]. При анкетировании больных СД2 в Москве низкоэнергетические переломы в анамнезе были выявлены в 30\% случаев [6], что почти в 2 раза превышает среднюю распространенность переломов в российской популяции [7]. Увеличение риска переломов у больных СД2 связывают с нарушением процесса костного ремоделирования в условиях гипергликемии и связанных с ней метаболических и гормональных расстройств [8].

Важную роль в ремоделировании костной ткани играют цитокины: фактор некроза опухоли а (TNF-a), интерлейкины1 $\beta,-4,-6,-10$ (IL-1 $\beta$, IL-4, IL-6, IL-10), фактор роста эндотелия сосудов (VEGF) и другие [9]. Цитокины оказывают прямое влияние на костную ткань, регулируя процессы пролиферации и дифференцировки остеобластов и остеокластов. Непрямое влияние цитокинов на ремоделирование кости опосредовано через изменения секреции лептина, адипонектина и других гормонов [10]. Увеличение продукции провоспалительных цитокинов вносит существенный вклад в развитие остеопороза при воспалительных заболеваниях [9]. Роль цитокинов в формировании остеопороза на фоне СД мало изучена. При этом известно, что при СД2 наблюдается дисбаланс в продукции провоспалительных и противовоспалительных цитокинов, отражающий развитие хронического воспаления низкой интенсивности $[11,12]$.

Ферменты из группы матриксных металлопротеиназ (MMPs: MMP-2, MMP-3, MMP-9 и другие) играют важную роль в обмене внеклеточного матрикса, формировании и ремоделировании кости [13]. Данные ферменты участвуют в рекрутировании остеокластов и реализации их функциональной активности, ангиогенезе, регуляции жизненного цикла остеоцитов. В связи с этим изменения продукции MMPs могут менять биомеханические свойства («качество») костной ткани [14].

Аллельные варианты генов цитокинов и MMPs определяют особенности продукции этих биологически активных молекул в нормальных и патологических условиях. В промоторах генов цитокинов и MMPs обнаружены полиморфные локусы, варианты нуклеотидов в которых влияют на уровень экспрессии генных продуктов $[15,16]$. У больных СД2 выявлены связи между однонуклеотидными полиморфизмами генов TNFA, IL4, IL6, IL10, VEGFA и уровнем соответствующих цитокинов в крови $[17,18]$. Роль вариативности в регуляторных участках генов цитокинов и MMPs в формировании индивидуальной предрасположенности к остеопорозу у больных СД2 не изучена.

\section{ЦЕЛЬ}

Определить ассоциации вариантов генотипов в промоторах генов цитокинов (TNFA, IL1B, IL4, IL6, IL10, VEGFA) и MMPs (MMP2, MMP3, MMP9) со снижением МПК у женщин с СД2, находящихся в постменопаузе.

\section{МЕТОДЫ}

\section{Дизайн исследования}

В данном одномоментном сравнительном исследовании тестировалась гипотеза о влиянии полиморфных вариантов промоторов генов провоспалительных и противовоспалительных цитокинов, а также ферментов группы MMPs на риск развития остеопении и остеопороза уженщин с СД2, находящихся в постменопаузе. Набор пациентов в исследование осуществлялся в одном клиническом центре в соответствии с критериями включения и исключения, установленными для гомогенизации выборки (см. ниже). После подписания информированного согласия у всех пациентов проводилось общеклиническое обследование и двухэнергетическая рентгеновская абсорбциометрия, по результатам которой больных делили на группы с нормальной и сниженной МПК. Гипо- 
теза исследования проверялась на основании оценки различий в частоте встречаемости аллелей и генотипов в полиморфных позициях промоторов генов цитокинов и MMPs, а также комбинаций этих генотипов, между группами больных с нормальной и сниженной МПК.

\section{Критерии соответствия}

Критериями включения в исследование являлись: женский пол, европеоидное происхождение, возраст от 50 до 70 лет, продолжительность менопаузы не менее одного года; диагноз СД2, установленный по критериям ВО3 (1999 г.) не менее пяти лет назад.

Основными критериями исключения являлись факторы риска развития вторичного остеопороза: заболевания эндокринной системы (гиперкортицизм, тиреотоксикоз, гипопитуитаризм, полигландулярные синдромы), системные ревматические заболевания (ревматоидный артрит, анкилозирующий спондилоартрит, другие диффузные болезни соединительной ткани), заболевания органов пищеварения, сопровождающиеся нарушением всасывания кальция (синдром мальабсорбции, состояния после резекции желудка, шунтирующих операций на желудочно-кишечном тракте и др.), заболевания почек недиабетической природы, хроническая болезнь почек (ХБП) 4-5-й стадии или трансплантация почек в анамнезе, заболевания системы крови (кроме железодефицитной анемии легкой степени), онкологические заболевания. В исследование не включались женщины, получающие заместительную постменопаузальную терапию, а также женщины с анамнестическими указаниями на прием глюкокортикоидов, иммуносупрессантов, тиазолидиндионов, ингибиторов обратной реабсорбции глюкозы в почках и препаратов для лечения остеопороза.

\section{Условия проведения}

Набор пациентов осуществлялся в клинике Научно-исследовательского института клинической и экспериментальной лимфологии - филиала ФГБНУ «Федеральный исследовательский центр Институт цитологии и генетики Сибирского отделения Российской академии наук» (г. Новосибирск, Россия).

\section{Описание медицинского вмешательства}

Исследование МПК проводили с помощью двухэнергетической рентгеновской абсорбциометрии на аппарате Lunar Prodigy (GE, CША). Определяли показатели МПК и Т-критерий в позвоночнике (поясничные позвонки: L1-L4), проксимальных отделах бедра, шейке бедра, предплечье недоминантной руки.

Определение риска основных низкоэнергетических переломов проводилось с помощью опросника FRAX (веб-версия 3.8, расчет по российской модели). Оценивали общий риск низкоэнергетических переломов (FRAX total) и риск переломов шейки бедра (FRAX hip).

Типирование аллельных вариантов в полиморфных позициях промоторов генов TNFA: -238 G/A(rs361525), -308 G/A (rs1800629), -863 C/A (rs1800630), IL1B: -31 T/C (rs1143627), IL4:-590C/T(rs 2243250), IL6:-174G/C(rs1800795), IL10: -592 C/A (rs1800872), -1082 A/G (rs1800896), VEGFA: -2578 A/C (rs699947), +936 C/T (rs3025039), MMP2: - 1306 T/C (rs243865), МMP3 -1171 5A/6A (rs35068180) и MMP9: -1562
C/T (rs34016235) осуществляли методом полимеразной цепной реакции в режиме реального времени с использованием коммерческих тест-систем на основе интеркалирующего красителя SYBR Greenl («Литех», Россия) и методом TaqMan зондов («Синтол», Россия) на амплификаторе «ДТ-96» («ДНК-Технология») согласно инструкциям фирм-производителей.

\section{Анализ в подгруппах}

На основании результатов двухэнергетической рентгеновской абсорбциометрии пациентки распределялись на группы с нормальной МПК, остеопенией и остеопорозом. При анализе данных молекулярно-генетического исследования женщины с остеопенией и остеопорозом рассматривались как группа со сниженной МПК.

\section{Основной исход исследования}

Основным показателем, оценивавшимся в исследовании, явились различия в частоте встречаемости аллелей и генотипов в полиморфных позициях промоторов генов цитокинов (TNFA, IL1B, IL4, IL6, IL 10, VEGFA) и MMPs (MMP2, MMP3, MМР9) в группах женщин в постменопаузе с СД2 с нормальной и сниженной МПК.

\section{Дополнительные исходы исследования}

Дополнительными показателями, оценивавшимися в исследовании, явились различия в частоте встречаемости комбинаций генотипов в полиморфных позициях промоторов генов цитокинов (TNFA, IL1B, IL4, IL6, IL10, VEGFA) и MMPs (MMP2, MMP3, MMP9) в группах женщин в постменопаузе с СД2 с нормальной и сниженной МПК.

\section{Этическая экспертиза}

Проведение исследования одобрено локальным Этическим комитетом ФГБНУ Научно-исследовательский институт клинической и экспериментальной лимфологии (протокол №104 от 20.10.2014). Все обследованные давали письменное информированное согласие на участие в исследовании.

\section{Статистический анализ}

Принципы расчета размера выборки

Размер выборки оценивали, исходя из ожиданий минимальной доли объясненной дисперсии полиморфизмов в 4\%. Тогда для ожидаемой мощности анализа в $80 \%$ при уровне значимости 0,05 необходимый размер выборки равен 200. Мы пользовались стандартной процедурой вычисления мощности анализа, которая предполагает, что тестовая статистика при альтернативной гипотезе имеет распределение Хи-квадрат с одной степенью свободы и заданным параметром нецентральности NCP, равным NCP=R2*N, где R2 - это ожидаемая доля объясненной дисперсии; N - размер выборки. Ожидаемую мощность задали равной $80 \%$, а порог значимости - 0,05.

\section{Memoды статистического анализа данных}

Статистическая обработка проведена с использованием программы STATISTICA 10 (StatSoft, Inc, 2011, (ША) и открытой программной среды R [19]. Учитывая, что распределение большинства изученных признаков было отличным от нормального, применяли методы непараметрической статистики. Межгрупповые различия 
Таблица 1. Клинико-лабораторная характеристика женщин с СД2, имеющих различную МПК

\begin{tabular}{|c|c|c|c|}
\hline \multirow{2}{*}{ Параметр } & \multicolumn{3}{|c|}{ МПк } \\
\hline & Норма $(\mathrm{n}=73)$ & Остеопения $(\mathrm{n}=90)$ & Остеопороз $(\mathrm{n}=34)$ \\
\hline Возраст, лет & $60(56 ; 64)$ & $64(60 ; 68)^{*}$ & $67(65 ; 69)^{* *}$ \\
\hline Длительность постменопаузы, лет & $11(6 ; 15)$ & $14,5(11 ; 20)^{*}$ & $17(13,5 ; 20)^{* *}$ \\
\hline Возраст наступления менопаузы, лет & $50(47 ; 52)$ & $49(45 ; 51)$ & $50(46 ; 52)$ \\
\hline Длительность СД2, лет & $14(10 ; 18)$ & $14(8 ; 19)$ & $11,5(9 ; 20)$ \\
\hline PoCT, $\mathrm{CM}$ & $160(157 ; 164)$ & $158(155 ; 163)$ & $157(153 ; 159)^{*}$ \\
\hline Масса тела, кг & $90(79 ; 100)$ & $85(74 ; 95)$ & $79(70 ; 89)^{* *}$ \\
\hline ИМТ, кг/м² & $35(31,2 ; 38,2)$ & $32,9(28,9 ; 37)$ & $33(28,5 ; 36)^{*}$ \\
\hline Соотношение ОТ/ОБ & $0,9(0,9 ; 1,0)$ & $1,0(0,9 ; 1,0)$ & $0,9(0,9 ; 1,0)$ \\
\hline $\mathrm{HbA}_{1 c^{\prime}} \%$ & $8,3(6,7 ; 10,1)$ & $8,2(7,0 ; 9,2)$ & $7,6(6,7 ; 9,0)^{*}$ \\
\hline Холестерин, ммоль/л & $5,0(4,5 ; 5,9)$ & $5,2(4,4 ; 6,1)$ & $5,4(4,5 ; 6,7)$ \\
\hline Триглицериды, ммоль/л & $1,7(1,2 ; 2,5)$ & $1,5(1,2 ; 1,9)$ & $1,9(1,5 ; 2,8)$ \\
\hline Холестерин ЛПНП, ммоль/л & $3,0(2,2 ; 3,8)$ & $3,0(2,3 ; 3,7)$ & $3,1(2,5 ; 3,7)$ \\
\hline Холестерин ЛПВП, ммоль/л & $1,2(1,0 ; 1,5)$ & $1,3(1,2 ; 1,6)$ & $1,3(1,0 ; 1,5)$ \\
\hline СКФ, мл/мин/1,73 м² & $68(59 ; 76)$ & $64(54 ; 76)$ & $77(58 ; 87)$ \\
\hline FRAX total & $6,3(5,8 ; 6,8)$ & $8,1(7,5 ; 9,8)^{*}$ & $9,4(8,4 ; 11)^{* *}$ \\
\hline FRAX hip & $0,1(0,1 ; 0,2)$ & $0,6(0,4 ; 1,0)^{*}$ & $1,1(0,6 ; 1,7)^{* *}$ \\
\hline
\end{tabular}

Примечания: данные представлены как медианы, 25-е; 75-е процентили; звездочка - статистически значимое (р<0,05) отличие: одна - с группой больных с нормальной МПК, две - с группой больных с нормальной МПК и остеопенией. Расчет СКФ осуществляли на основе уровня креатинина в сыворотке крови по формуле CKD-EPI (2009 г.), у лиц с морбидным ожирением - по пробе Реберга-Тареева.

оценивали с помощью критерия Манна-Уитни и ANOVA Краскела-Уоллиса. При статистическом анализе результатов генетического исследования рассчитывали частоту встречаемости аллелей и генотипов, отношение шансов (ОШ), 95\% доверительный интервал $(95 \%$ CI), специфичность (Sp). Распределение генотипов по исследованным полиморфным локусам проверяли на соответствие равновесию Харди-Вайнберга (РХВ). Достоверность различий частот распределения изучаемых признаков в альтернативных группах определяли по критерию $X^{2}$ с поправкой Йетса на непрерывность и двустороннему варианту точного метода Фишера для четырехпольных таблиц. При анализе использованы аддитивная, рецессивная, доминантная и сверхдоминантная модели наследования. Взаимодействие между генами оценивалось по стандартной модели взаимодействия $\mathrm{Y}$ b1*SNP1+b2 *SNP2+b12*SNP1*SNP2, где определялась значимость отличия эффекта взаимодействия b12 от нуля. Взаимосвязь клинических и генетических признаков со снижением МПК проверяли в моделях однофакторного и многофакторного регрессионного анализа. Критический уровень значимости при проверке статистических гипотез принимали равным 0,05, для результатов биоинформационного анализа комбинаций полиморфизмов - 0,005.

\section{РЕЗУЛЬТАТЫ}

Объекты (участники) исследования

В исследование включены 197 женщин с СД2, считающих себя и своих родителей русскими. Индекс массы тела

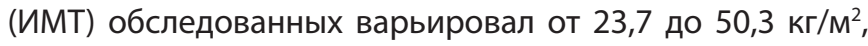
составляя в среднем 33,3 кг/м². Избыточную массу тела имели 48 женщин, ожирение - 146. О курении сигарет сообщили 6 женщин. Низкоэнергетические переломы в анамнезе зарегистрированы у 25 женщин, у 148 переломы отсутствовали, данные о переломах у 24 больных не получены. Длительность СД2 варьировала от 5 до 36 лет (медиана 14,3 года). Диабетическая ретинопатия выявлена у 132 обследованных, поражения крупных сосудов у 129. Диабетическая нефропатия имелась у 148 пациенток, из них у 27 диагностирована хроническая болезнь почек (ХБП) 1-й стадии, у 73 - 2-й стадии, у 39 - ХБП стадии ЗА, у девяти - стадии ЗБ. Все обследованные получали сахароснижающую терапию. Лечение инсулином получали 144 женщины, из них 82 - в сочетании с метформином, 5 с препаратами сульфонилмочевины (ПСМ), 17 - с метформином и ПСМ. Шестнадцать пациенток принимали метформин в виде монотерапии, 8 принимали только ПСМ, 29 получали комбинацию метформина и ПСМ. Медиана уровня гликированного гемоглобина $\mathrm{A}_{1 c}\left(\mathrm{HbA}_{1 c}\right)$ составила 8,2\% (25-75 процентиль: 6,8-9,5\%).

На основании наименьшего Т-критерия пациентки разделены на группы с нормальной МПК $(n=73)$, остеопенией $(n=90)$ и остеопорозом $(n=34)$. Женщины, имеющие остеопороз, в сравнении с женщинами с нормальной МПК были старше, ниже ростом, имели меньшую массу тела и большую длительность постменопаузы (табл. 1). Уровень $\mathrm{HbA}_{1 c}$ оказался ниже у женщин с остеопорозом в сравнении с пациентками с нормальной МПК. Не выявлено достоверных отличий между группами по соотношению окружности талии к окружности бедер (ОТ/ОБ), длительности СД2, уровню липидов, скорости клубочковой фильтрации (СКФ). Показатели FRAX ожидаемо оказались выше у лиц с остеопенией и остеопорозом. 
Таблица 2. Ассоциации полиморфизмов генов цитокинов и MMPs с Т-критерием у женщин с СД2

\begin{tabular}{|c|c|c|c|c|c|c|c|}
\hline \multirow[b]{2}{*}{ Полиморфизм } & \multirow[b]{2}{*}{ Частота } & \multirow[b]{2}{*}{$\begin{array}{c}\text { Отклонение } \\
\text { от РXВ }\end{array}$} & \multirow[b]{2}{*}{$\begin{array}{c}\text { Эффекторный } \\
\text { аллель }\end{array}$} & \multirow[b]{2}{*}{$\begin{array}{l}\text { Другой } \\
\text { аллель }\end{array}$} & \multicolumn{3}{|c|}{ Т-критерий } \\
\hline & & & & & Эффект & $\begin{array}{l}\text { Станд. } \\
\text { ошибка }\end{array}$ & $\mathrm{P}$ \\
\hline TNFA -238 G/A & 0,07 & 0,28 & $A$ & $G$ & $-0,15$ & 0,197 & 0,44 \\
\hline TNFA $-308 \mathrm{G} / \mathrm{A}$ & 0,16 & 0,12 & $A$ & $G$ & 0,11 & 0,154 & 0,49 \\
\hline TNFA $-863 C / A$ & 0,16 & 0,35 & $A$ & $C$ & 0,04 & 0,141 & 0,79 \\
\hline IL1B-31T/C & 0,38 & 0,34 & $C$ & $T$ & $-0,09$ & 0,106 & 0,37 \\
\hline IL4 -590 C/T & 0,76 & 0,89 & $C$ & $T$ & 0,00 & 0,123 & 0,98 \\
\hline IL6-174 G/C & 0,50 & 0,57 & $C$ & G & 0,09 & 0,108 & 0,38 \\
\hline IL10-592C/A & 0,24 & 0,29 & $A$ & $C$ & 0,02 & 0,120 & 0,9 \\
\hline IL $10-1082 \mathrm{~A} / \mathrm{G}$ & 0,55 & 0,01 & $A$ & $G$ & 0,03 & 0,097 & 0,72 \\
\hline$V E G F A+936 C / T$ & 0,82 & 0,28 & $C$ & $T$ & $-0,12$ & 0,165 & 0,46 \\
\hline VEGFA $-2578 C / A$ & 0,47 & 0,51 & $A$ & $C$ & $-0,06$ & 0,103 & 0,54 \\
\hline MMP2 -1306 T/C & 0,68 & 0,0000005 & $C$ & $T$ & $-0,01$ & 0,098 & 0,9 \\
\hline MMP3 - $11715 A / 6 A$ & 0,45 & 0,69 & $5 A$ & $6 A$ & 0,14 & 0,106 & 0,19 \\
\hline$M M P 9-1562 C / T$ & 0,80 & 0,07 & $C$ & $T$ & $-0,39$ & 0,121 & 0,001 \\
\hline
\end{tabular}

Примечание: РХВ - равновесие Харди-Вайнберга.

Таблица 3. Комбинации полиморфизмов генов цитокинов и MMPs, положительно ассоциированные со снижением МПК, у женщин с СД2

\begin{tabular}{lccccc}
\hline \multicolumn{1}{c}{ Комбинация полиморфизмов } & Генотип & ош & $\mathbf{9 5 \%}$ ди & $\mathbf{P}$ & Sp \\
\hline TNFA -863:MMP9-1562 & CC-CC & 2,04 & $1,08-3,86$ & 0,001 & 69,12 \\
TNFA -308:MMP9-1562 & $G G-C C$ & 2,4 & $1,28-4,52$ & 0,001 & 66,18 \\
IL1B-31:IL6-174 & TC-GC & 2,27 & $1,03-5,03$ & 0,002 & 84,62 \\
IL10-1082:MMP2-1306 & AA-CC & 2,58 & $1,09-6,07$ & 0,003 & 87,10 \\
VEGFA -2578:MMP9-1562 & AA-CC & 3,4 & $1,10-10,48$ & 0,004 & 94,03 \\
TNFA -863:TNF-308:MMP9-1562 & CC-GG-CC & 2,24 & $1,08-4,61$ & 0,004 & 80,88 \\
TNFA -863:IL4-590:MMP3-1171 & CC-CT-5A6A & 3,67 & $1,19-11,32$ & 0,004 & 93,55 \\
TNFA -863:IL10-1082:MMP2-1306 & CC-AA-CC & 3,02 & $1,08-8,45$ & 0,004 & 91,94 \\
TNFA -863:VEGFA +936:MMP9-1562 & CC-CC-CC & 2,92 & $1,22-6,95$ & 0,005 & 84,31 \\
TNFA -308:TNFA -238:MMP9-1562 & GG-GG-CC & 1,96 & $1,04-3,72$ & 0,005 & 69,12 \\
TNFA -308:IL10-1082:MMP2-1306 & GG-AA-CC & 2,85 & $1,02-8,00$ & 0,005 & 91,94 \\
TNFA -308:IL10-1082:MMP9-1562 & GG-GG-CC & 4,21 & $1,19-14,91$ & 0,005 & 95,31 \\
\hline
\end{tabular}

\section{Основные результаты исследования}

В однофакторном регрессионном анализе значимую ассоциацию с МПК показали возраст $(p<0,0001)$, рост $(p<0,0001)$, масса тела $(p<0,0001)$, ИМТ $(p=0,002)$, длительность менопаузы ( $p=0,0001)$, курение $(p=0,008)$. В многофакторной модели наиболее значимо с МПК были ассоциированы возраст ( $p<0,0001)$, ИМТ $(p=0,003)$ и курение $(p=0,01)$, которые в сумме объясняли $21,6 \%$ общей дисперсии Т-критерия и далее использовались как ковариаты для анализа ассоциаций с генотипами.

Результаты анализа ассоциаций полиморфизмов генов цитокинов и MMPs c T-критерием представлены в табл. 2. В аддитивной модели установлена связь аллеля $C$ и гомозиготного генотипа СС в позиции -1562 гена MMP9 с низкой МПК (ОШ 2,16, 95\% ДИ 1,29-3,6, p=0,0007 и ОШ 2,02, 95\% ДИ 1,08-3,79, p=0,0008 соответственно). Мы исследовали также неаддитивные модели наследования (рецессивная, доминантная и сверхдоминантная), однако анализ не выявил дополнительных значимых сигналов. При использовании клинических параметров в качестве ковариатов и аддитивной модели наследования показана достоверная ассоциация полиморфизма в гене MMP9 - 1562 C/T ( $<<0,001)$ с МПК, объясняющая дополнительно 4\% общей дисперсии Т-критерия.

Дополнительные результаты исследования

При биоинформационном анализе выявлено 12 комбинаций генотипов, положительно ассоциированных с низкой МПК с вероятностью $\mathrm{p}<0,005$ (табл. 3). В составе этих комбинаций наиболее часто встречался гомозиготный генотип СС в позиции -1562 гена MMP9 (7 комбинаций), генотип СС в позиции -863 гена TNFA (5 комбинаций), генотип GG в позиции -308 гена TNFA (4 комбинации) и генотип $A A$ в позиции -1082 гена IL10 (3 комбинации). Особенностью выделенных комбинированных признаков являлась высокая специфичность ассоциации с изучае- 
мой патологией: они практически не встречались в группе больных с нормальной МПК.

Нежелательные явления

В ходе исследования каких-либо нежелательных явлений не зафиксировано.

\section{ОБСУЖДЕНИЕ}

\section{Резюме основного результата исследования}

Представленное исследование показало независимую ассоциацию между низкой МПК и носительством генотипа СС в позиции -1562 промотора гена MMP9 у женщин с СД2 в постменопаузе.

\section{Обсуждение основного результата исследования}

Известно, что МПК человека в значительной степени детерминирована генетически [20]. В последние годы накоплены данные об ассоциациях остеопороза с большим количеством полиморфных вариантов генов. Так, база данных Public Health Genomics Knowledge Base содержит ссылки на исследования ассоциаций 180 генов (по состоянию на 18.07.2017) с постменопаузальным остеопорозом [21]. Наиболее обсуждаемыми являются гены рецептора витамина D (VRD), рецепторов эстрогенов (ESR1, ESR2), а1-цепи коллагена I типа (COL1A1), остеопротегерина (TNFRSF11B), IL-6 (IL6), метилентетрагидрофолатредуктазы (MTHFR), белка 5 типа, связанного с рецептором липопротеинов низкой плотности (LRP5). Полногеномные исследования выявили дополнительные полиморфные локусы, ассоциированные с МПК, качеством кости и/или риском переломов. Влияние этих локусов может реализоваться через связывание транскрипционных факторов, микроРНК, активность сигнального пути Wnt и других внутриклеточных путей передачи сигнала, дифференцировку стволовых мезенхимальных клеток и остеоцитов [22, 23].

Роль генетических факторов в развитии остеопороза на фоне СД2 остается малоизученной. В данном исследовании мы тестировали гипотезу о связи полиморфных вариантов промоторов генов цитокинов и MMPs со снижением МПК у женщин с СД2 в постменопаузе. Исследовав 13 точек полиморфизма в регуляторных областях 9 генов, мы выявили более высокую распространенность аллеля С и гомозиготного генотипа СС в позиции -1562 гена MMP9 у женщин с остеопенией и остеопорозом. Выявленная ассоциация сохраняла свою значимость после учета клинических факторов риска, таких как возраст, ИМТ и курение. Насколько нам известно, связь полиморфизма гена MMP9 с остеопорозом зафиксирована впервые. При этом компьютерный анализ (text mining) сетевых генно-белковых взаимодействий позиционирует ген MMP9 в составе генных кластеров, ассоциированных с остеопорозом [24]. Описана обратная взаимосвязь между уровнем ММР-9 и МПК у больных с хронической обструктивной болезнью легких [25]. Первоначально роль MMPs в биологии костной ткани связывали со способностью этих ферментов разрушать коллаген. В дальнейшем было показано, что MMPs участвуют в межклеточных и клеточно-матриксных взаимодействиях, выполняя функции важных регуляторов в формировании, оссификации и постнатальном ремоделировании костей [26].
Взятый как отдельный признак, В совокупности с «традиционными» клиническими факторами риска, полиморфизм МMP9 позволял объяснить относительно небольшую часть дисперсии Т-критерия (около 25\%). Одним из способов повышения прогностической значимости генетических признаков является поиск комбинаций генов (так называемых генных сетей), ассоциированных с развитием патологии. Этот подход все чаще используется для изучения наследственной основы мультифакториальных заболеваний человека, в том числе и СД $[27,28]$. Показано, что больные СД2 отличаются от общей популяции структурой генных сетей цитокинов и MMPs $[29,30]$. С помощью биоинформационного анализа мы выявили ряд комбинаций генотипов цитокинов и MMPs, accoциированных со снижением МПК. Особенностью этих комбинаций оказалась необычно высокая частота гомозиготных генотипов в полиморфных позициях промоторов генов TNFA (генотип CС в позиции -863 и генотип GG в позиции -308) и IL10 (генотип $A A$ в позиции -1082). Достаточно высокая сила ассоциации комбинированных генетических признаков со снижением МПК (показатели отношения шансов и специфичности) позволяет рассматривать эти признаки как потенциальные маркеры, пригодные для персонифицированного прогноза.

\section{Ограничения исследования}

Одномоментный дизайн не позволяет судить о причинно-следственных взаимосвязях между признаками. Относительно небольшой объем выборки и низкая распространенность комбинаций генотипов в обследованных группах требуют осторожности при интерпретации данных биоинформационного анализа.

\section{ЗАКЛЮЧЕНИЕ}

Снижение МПК у женщин с СД2, находящихся в постменопаузе, ассоциировано с увеличением возраста, уменьшением ИМТ, курением. Наличие аллеля С и генотипа СС в полиморфной позиции -1562 промотора гена MMP9(rs34016235) увеличивает вероятность снижения МПК, в дополнение к клиническим факторам риска. В составе генетических комбинаций, ассоциированных со снижением МПК у женщин с СД2, преобладают гомозиготные варианты в полиморфных позициях промоторов генов: генотип СС в позиции -1562 гена MMP9, генотип CC в позиции -863 гена TNFA, генотип GG в позиции -308 гена TNFA и генотип AA в позиции -1082 гена IL 10. Поскольку указанные варианты связаны с различной интенсивностью транскрипции генов, можно предполагать их патогенетическую связь с формированием остеопении и остеопороза. Полученные данные указывают на перспективность анализа генных сетей факторов, вовлеченных в ремоделирование костной ткани, для поиска персонифицированных предикторов остеопороза при СД.

\section{ДОПОЛНИТЕЛЬНАЯ ИНФОРМАЦИЯ}

Источник финансирования. Работа выполнена за счет средств государственного задания Научно-исследовательского института клинической и экспериментальной лимфологии - филиала ФГБНУ «Федеральный исследовательский центр Институт цитологии и генетики Сибирского отделения Российской академии наук». 
Конфликт интересов. Авторы декларируют отсутствие явных и потенциальных конфликтов интересов, связанных с публикацией настоящей статьи.

Участие авторов. Фазуллина О.Н. - сбор клинического материала, выполнение денситометрии, статистическая обработка результатов, написание текста; Климонтов В.В. - концепция и дизайн исследования, анализ данных, написание текста; Коненков В.И. - концепция и дизайн исследования, анализ данных; Шевченко А.В. - выполнение молекулярно-генетических исследований; Прокофьев В.Ф., Цепилов Я.А. - статистическая обработка результатов.

\section{СПИСОК ЛИТЕРАТУРЫ | REFERENCES}

1. Дедов И.И., Мельниченко Г.А., Белая Ж.Е., и др. Остеопороз от редкого симптома эндокринных болезней до безмолвной эпидемии XX-XXI века // Проблемы эндокринологии. - 2011. T. 57. - №1. - C. 35-45. [Dedov II, Mel'nichenko GA, Belaia ZE, et al. Osteoporosis: from a rare symptom of endocrine diseases to the tacit epidemic of XX-XXI centuries. Problems of Endocrinology. 2011;57(1):35-45 (In Russ.)] doi: 10.14341/probl201157135-45

2. Молитвословова Н.А., Галстян Г.Р. Остеопороз и сахарный диабет: современный взгляд на проблему // Сахарный диабет. - 2013. T. 16. - №1. - C. 57-62. [Molitvoslovova NA, Galstyan GR. Osteoporosis and diabetes mellitus: a modern viewpoint. Diabetes Mellitus. 2013;16(1):57-62. (In Russ.)] doi: 10.14341/2072-0351-3598

3. Rathmann W, Kostev K. Fracture risk in patients with newly diagnosed type 2 diabetes: a retrospective database analysis in primary care. J Diabetes Complications. 2015;29(6):766-770. doi: 10.1016/j.jdiacomp.2015.05.007

4. Kim SH, Kim YM, Yoo JS, et al. Increased risk of hip fractures in Korean patients with type 2 diabetes: a 6-year nationwide population-based study. J Bone Miner Metab. 2016;35(6):623-629. doi: 10.1007/s00774-016-0798-z

5. Schwartz AV, Vittinghoff E, Bauer DC, et al. Association of BMD and FRAX score with risk of fracture in older adults with type 2 diabetes. JAMA. 2011;305(21):2184-2192. doi: 10.1001/jama.2011.715

6. Ялочкина Т.О., Белая Ж.Е., Рожинская Л.Я., и др. Переломы костей при сахарном диабете 2 типа: распространенность и факторы ри ска // Сахарный диабет. - 2016. - Т. 19. - №5. - C. 359-365. [Yalochkina TO, Belaya ZE, Rozhnskaya LY, et al. Bone fractures in patients with type 2 diabetes mellitus: Prevalence and risk factors. Diabetes mellitus. 2016;19(5):359-365. (In Russ.)] doi: 10.14341/DM7796

7. Андреева Т.М. Травматизм в Российской Федерации на основе данных статистики // Социальные аспекты здоровья населения. 2010. - T. 16. - №4. - C. 2. [Andreeva TM. Traumatism in the Russian Federation on the basis of statistics data. Sotsial'nye aspekty zdorov'ya naseleniya. 2010;16(4):2. (In Russ.)]

8. Farr JN, Khosla S. Determinants of bone strength and quality in diabetes mellitus in humans. Bone. 2016;82:28-34 doi: 10.1016/j.bone.2015.07.027

9. Pietschmann P, Mechtcheriakova D, Meshcheryakova A, et al. Immunology of Osteoporosis: A Mini-Review. Gerontology. 2016;62(2):128-137. doi: 10.1159/000431091

10. Wang T, He C, Yu X. Pro-Inflammatory Cytokines: New Potential Therapeutic Targets for Obesity-Related Bone Disorders. Curr Drug Targets. 2017;18(14):1664-1675. doi: 10.2174/1389450118666170104153512

11. Pirola $L$, Ferraz JC. Role of pro- and anti-inflammatory phenomena in the physiopathology of type 2 diabetes and obesity. World J Biol Chem. 2017;8(2):120-128. doi: 10.4331/wjbc.v8.i2.120

12. Коненков В.И., Климонтов В.В., Мякина Н.Е., и др. Повышенная концентрация воспалительных цитокинов в сыворотке крови у больных сахарным диабетом 2-го типа с хронической болезнью почек // Терапевтический архив. - 2015. - Т. 87. - №6. - C. 45-49. [Konenkov VI, Klimontov W, Myakina NE, et al. Increased serum concentrations of inflammatory cytokines in type 2 diabetic patients with chronic kidney disease. Ter Arkh. 2015;87(6):45-49. (In Russ.)] doi: 10.17116/terarkh201587645-49

13. Paiva KBS, Granjeiro JM. Matrix Metalloproteinases in Bone Resorption, Remodeling, and Repair. Prog Mol Biol Trans/ Sci. 2017;148:203-303. doi: 10.1016/bs.pmbts.2017.05.001

14. Paiva KB, Granjeiro JM. Bone tissue remodeling and development: focus on matrix metalloproteinase functions. Arch Biochem Biophys. 2014;561:74-87. doi: 10.1016/j.abb.2014.07.034

15. Шевченко А.В., Коненков В.И., Прокофьев В.Ф., и др. Конституциональные основы уровней спонтанной и индуцированной продукции цитокинов TNA-A, IL-1ß, IL-4, IL-6 и IL-10 у здоровых лиц европеоидного населения России // Иммунология. - 2016. - Т. 37. №5. - C. 232-238. [Shevchenko AV, Konenkov VI, Prokofiev VF, et al. Con- stitutional basis of the levels of spontaneous and induced production of cytokines TNA-a, IL-1 $\beta, I L-4, I L-6$ and IL-10 in healthy individuals of the european population of Russia. Immunologiia. 2016;37(5):232-238. (In Russ.)] doi: 10.18821/0206-4952-2016-37-5-232-238

16. Papageorgiou N, Tousoulis D, Hatzis G, et al. Genetic Variability of Matrix Metalloproteinase Genes in Cardiovascular Disease. Curr Top Med Chem. 2012;12(10):1206-1213. doi: 10.2174/1568026611208011206

17. Tyan NV, Klimontov WV, Shevchenko AV, et al. Polymorphisms in the gene promoters of IL4, IL6, IL10 and TNFA associated with serum levels of cytokines in type 2 diabetic subjects. Diabetologia. 2016:59(S1):513.

18. К Климонтов В.В, Тян Н.В., Орлов Н.Б., и др. Взаимосвязь уровня фактора роста эндотелия сосудов в сыворотке крови и полиморфизма гена VEGFA с ишемической болезнью сердца у больных сахарным диабетом 2 типа // Кардиология. - 2017. - Т. 57. - №5. C. 17-22. [Klimontov WV, Tyan NV, Orlov NB, et al. Association of serum levels and gene polymorphism of vascular endothelium growth factor with coronary artery disease in type 2 diabetic patients. Cardiology. 2017;57(5):17-22. (In Russ.)] doi: 10.18565/cardio.2017.5.17-22

19. Ihaka R, Gentleman R. R: A Language for Data Analysis and Graphics. J Comput Graph Stat. 1996;5(3):299-314. doi: 10.1080/10618600.1996.10474713

20. Medina-Gomez C, Chesi A, Heppe DH, et al. BMD Loci Contribute to Ethnic and Developmental Differences in Skeletal Fragility across Populations: Assessment of Evolutionary Selection Pressures. Mol Biol Evol. 2015;32(11):2961-2972. doi: 10.1093/molbev/msv170

21. phgkb.cdc.gov [Internet]. Public Health Genomics Knowledge Base (v2.1). [updated 2018 Feb 23; cited 2018 Feb 25]. Available from: https://phgkb.cdc.gov/PHGKB/phenoPedia.action?firstQuery=Osteoporosis,Postmenopausal\&cuilD=C0029458\&typeSubmit=GO\&check=y\&which=2\&pubOrderType=pubD.

22. Hsu YH, Kiel DP. Clinical review: Genome-wide association studies of skeletal phenotypes: what we have learned and where we are headed. J Clin Endocrinol Metab. 2012;97(10):E1958-1977. doi: 10.1210/jc.2012-1890

23. Qin L, Liu Y, Wang Y, et al. Computational Characterization of Osteoporosis Associated SNPs and Genes Identified by Genome-Wide Association Studies. PLoS One. 2016;11(3):e0150070. doi: 10.1371/journal.pone.0150070

24. Toti P, Sbordone C, Martuscelli R, et al. Gene clustering analysis in human osteoporosis disease and modifications of the jawbone. Arch Oral Biol. 2013;58(8):912-929. doi: 10.1016/j.archoralbio.2013.02.013

25. Кочеткова Е.А., Угай Л.Г., Майстровская Ю.В., и др. Роль матриксной металлопротеиназы-9 в патогенезе остеопороза у пациентов с хронической обструктивной болезнью легких // Терапевтический архив. - 2012. - T. 84. - №8. - C. 37-40. [Kochetkova EA, Ugă̌ LG, Maistrovskaya YV, et al. Role of matrix metalloproteinase- 9 in the pathogenesis of osteoporosis in patients with chronic obstructive pulmonary disease. Ter Arkh. 2012;84(8):37-40. (In Russ.)]

26. Aiken A, Khokha R. Unraveling metalloproteinase function in skeletal biology and disease using genetically altered mice. Biochim Biophys Acta. 2010;1803(1):121-132. doi: 10.1016/j.bbamcr.2009.07.002

27. Winkler C, Krumsiek J, Lempainen J, et al. A strategy for combining minor genetic susceptibility genes to improve prediction of disease in type 1 diabetes. Genes Immun. 2012;13(7):549-555 doi: 10.1038/gene.2012.36

28. Knuppel S, Meidtner K, Arregui M, et al. Joint effect of unlinked genotypes: application to type 2 diabetes in the EPIC-Potsdam case-cohort study. Ann Hum Genet. 2015;79(4):253-263. doi: 10.1111/ahg.12115

29. Коненков В.И., Шевченко А.В., Прокофьев В.Ф., и др. Ассоциации вариантов гена фактора роста сосудистого эндотелия (VEGF) и генов цитокинов (IL1B, IL4, IL6, IL10, TNFA) с сахарным диабетом 2 типа у женщин // Сахарный диабет. - 2012. - T. 15. - №3. C. 4-10. [Konenkov VI, Shevchenko AV, Prokof'ev VF, et al. Associations 
of vascular endothelial growth factor (VEGF) gene and cytokine (IL1B, IL4, IL6, IL10, TNFA) genes combinations with type 2 diabetes mellitus in women. Diabetes Mellitus 2012;15(3):4-10. (In Russ.)] doi: 10.14341/2072-0351-6079

30. Коненков В.И., Шевченко А.В., Прокофьев В.Ф., и др. Генетические факторы индукции нарушений регуляции ангиоге- неза у больных сахарным диабетом 2 типа // Медицинская иммунология. - 2012. - Т. 14. - №6. - C. 489-500. [Konenkov VI, Shevchenko AV, Prokof'ev VF, et al. Genetic factors inducing disturbed regulation of angiogenesis in diabetes mellitus type 2 . Meditsinskaia immunologiia. 2012;14(6):489-500. (In Russ.)] doi: 10.15789/1563-0625-2012-6-489-500

\section{ИНФОРМАЦИЯ ОБ АВТОРАХ [AUTHORS INFO]}

Климонтов Вадим Валерьевич, д.м.Н., профессор [Vadim V. Klimontov, MD, PhD, Professor]; адрес: Россия, 630060, Новосибирск, ул. Тимакова, 2 [address: 2, Timakov str., Novosibirsk, 630060 Russian Federation]; ORCID: http://orcid.org/0000-0002-5407-8722; e-Library SPIN: 1734-4030; тел. раб.: +7 (383) 335-96-33; e-mail: klimontov@mail.ru.

Фазуллина Ольга Николаевна, врач-эндокринолог, м.н.с. [Olga N. Fazullina, MD, junior research associate]; ORCID: http://orcid.org/0000-0002-5868-579X; e-Library SPIN: 6055-5870; e-mail: fazullina@ngs.ru.

Коненков Владимир Иосифович, д.м.Н., профессор, академик PAH [Vladimir I. Konenkov, MD, PhD, Professor]; ORCID: http://orcid.org/0000-0001-7385-6270; eLibrary SPIN: 7822-9674; e-mail: vikonenkov@gmail.com.

Шевченко Алла Владимировна, д.б.н. [Alla V. Shevchenko, PhD in Biology];

ORCID: http://orcid.org/0000-0001-5898-950X; eLibrary SPIN: 7553-8222; e-mail: shalla64@mail.ru.

Прокофьев Виктор Федорович, к.м.н. [Viktor F. Prokoviev, MD, PhD]; ORCID: http://orcid.org/0000-0001-7290-1631; eLibrary SPIN: 2318-1500; e-mail: vprok@ngs.ru.

Цепилов Яков Александрович, к.б.н. [Yakov A. Tsepilov, PhD in Biology]; ORCID: http://orcid.org/0000-0002-4931-6052; eLibrary SPIN: 1647-0654; e-mail: tsepilov@bionet.nsc.ru.

\section{ЦИТИРОВАТЬ:}

Фазуллина О.Н., Климонтов В.В., Коненков В.И., Шевченко А.В., Прокофьев В.Ф., Цепилов Я.А. Ассоциации полиморфизмов генов цитокинов и матриксных металлопротеиназ с минеральной плотностью костной ткани у женщин в постменопаузе с сахарным диабетом 2 типа // Сахарный диабет. — 2018. - Т. 21. — №1 _ — С. 26-33. doi: 10.14341/DM8825

\section{TO CITE THIS ARTICLE:}

Fazullina ON, Klimontov VV, Konenkov VI, Shevchenko AV, Prokofiev VF, Tsepilov YA. Associations of polymorphisms in the gene promoters of cytokines and matrix metalloproteinases with bone mineral density in postmenopausal type 2 diabetic women. Diabetes mellitus. 2018;21(1):26-33. doi: 10.14341/DM8825 